\section{REVISTA INTERNACIONAL DE CIENCIAS DEL DEPORTE International Journal of Sport Science}

Rev. int. cienc. deporte

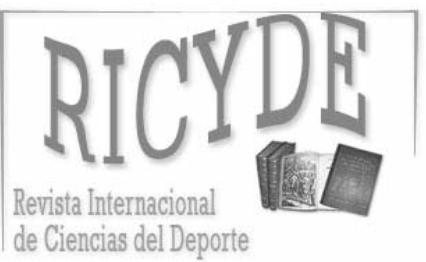

International Journal of Sport Science VOLUMEN VI - AÑO VI

Páginas:112-127 ISSN:1885-3137

No 19 - Abril - 2010

\title{
Sistema de codificación para el análisis de los indicadores de calidad de las cartas de servicios en materia deportiva. Coding system for the analysis of quality markers of service cards in sport.
}

\author{
Alberto Blázquez Manzano \\ Consejería de los Jóvenes y del Deporte. Junta de Extremadura. España \\ Sebastián Feu Molina \\ Facultad de Educación. Universidad de Extremadura. España
}

\section{Resumen}

El objetivo del estudio fue desarrollar un sistema de categorías para analizar el contenido de los indicadores de calidad de las cartas de servicio de las treinta Mancomunidades de Extremadura y del Ayuntamiento de Madrid. A través de un análisis deductivo - inductivo de los indicadores de calidad y de un proceso de concordancia consensuada intercodificadores, se construyó el sistema de codificación. El instrumento, se estructuró en tres dimensiones relativas a los indicadores de calidad: objeto (destinatarios), temática (asunto sobre el que trata) y temporalidad (momento en que se mide). La calidad de dato fue estudiada a través del porcentaje de acuerdo, de los coeficientes de correlación y del índice de Kappa de Cohen inter e intra codificador. Los resultados indican una adecuada calidad de dato y hacen que esta herramienta sea un instrumento útil para analizar la orientación de los indicadores de calidad de las cartas de servicio seleccionadas. La información, obtenida del análisis de los indicadores de calidad, facilitará la adaptación de las políticas deportivas a las demandas de la ciudadanía a través de las cartas de servicios mediante un mejor conocimiento de los elementos que conforman el sistema deportivo.

Palabras clave: cartas de servicio; indicadores de calidad; sistema deportivo; análisis de contenido; sistema de categorías; calidad de dato.

\begin{abstract}
The aim of the study was to develop a category system for analyzing the content of the markers of quality of service cards of the thirty Associations of Extremadura and Madrid City Council. Through a deductive analysis - inductive quality indicators and an agreed matching process was built inter-encoders coding system. The instrument was structured in three dimensions related to quality markers: object (target), subject (matter that is) and temporal (when measured). The quality of data was studied by the percentage of agreement, correlation coefficients and Cohen's Kappa index of inter-and intra encoder. The results indicate an adequate quality of data and make it be a useful tool for analyzing the orientation of the quality markers of service cards selected. The information gained from the analysis of quality markers will facilitate the adaptation of sports policies to the demands of citizenship through service cards.
\end{abstract}

Key words: service cards; quality markers; sport system; analysis of content; system of categories; quality of information. 
Blázquez, A.; Feu, S. (2010). Sistema de codificación para el análisis de los indicadores de calidad de las cartas de servicios en materia deportiva. Revista Internacional de Ciencias del Deporte. 19(6), 112-127. http://www.cafyd.com/REVISTA/01902.pdf

\section{Introducción}

U no de los principales problemas de la gestión en las entidades deportivas es que al ser sistemas abiertos donde confluyen multitud de agentes (patrocinadores, usuarios, medios de comunicación...) y temáticas (económica, marketing, etc.), se hace difícil su control y evaluación. Para lograr la máxima eficiencia en satisfacer las demandas de la ciudadanía, es fundamental desarrollar estrategias de segmentación que permitan conocer en profundidad el perfil de los clientes utilizando variables sociales, subjetivas y simbólicas (Luna-Arocas, 1998). Así, esta tendencia hacia lo específico se manifiesta en multitud de documentos que desarrollan la planificación de la calidad en el ámbito deportivo: planes estratégicos, cartas de servicios, planes directores de instalaciones deportivas, etc.

La estructura de los planes estratégicos suelen tener cierta similitud: diagnóstico general del entorno, análisis DAFO (debilidades, amenazas, fortalezas y oportunidades), misión, objetivos y líneas de actuación. El grado de concreción de los apartados anteriores facilita la implantación de las medidas adoptadas. Es frecuente realizar un diagnóstico de la situación focalizándola en diversos centros de interés donde se entremezclan elementos tangibles (ejemplo: instalaciones deportivas, recursos económicos) o intangibles (ejemplo: actividades, procesos.), con temáticas (salud, ocio, recreación, igualdad de oportunidades...); lo cual, dificulta ver con claridad los elementos básicos que componen el sistema deportivo y, por tanto, desarrollar una estrategia global que integre a los mismos.

Existen estudios relacionados con las diferentes áreas que desarrollan los gestores deportivos, entre los que destacan: la formación de los mismos (López, 2001), los modelos de gestión (González, 2004), las áreas de satisfacción de los usuarios con respecto a los servicios deportivos (Calabuig, Quintanilla y Mundina, 2008; Luna-Aroca y Mundina, 1998) o el análisis de áreas específicas tales como: instalaciones deportivas, recursos humanos (García, 2007), actividades deportivas (Gambau, 2004), gestión económica y financiera (Jiménez, Quesada, Aranguren, y Ortega, 2006), etc. Sin embargo, son escasos los estudios relacionados con la gestión deportiva que parten del análisis de los propios documentos de gestión tales como: cartas de servicio, planes estratégicos, etc.

\section{La calidad de los servicios y su aplicación a la actividad físico deportiva}

Las diferentes demandas que impone la realidad deportiva, requiere la necesidad de garantizar la continuidad de las entidades permitiendo su progreso y adaptación, de ahí que en los últimos años el concepto de calidad se considere como el mayor potencial referido a la superioridad competitiva que hoy en día pueden tener las empresas (Lloréns y Fuentes, 2000), y que en la actualidad afecta también al ámbito de las administraciones públicas.

Para entender mejor qué es la calidad, es preciso tener en cuenta dos conceptos clave: percepción y expectativas que tienen los clientes (externos e internos) con respecto al servicio recibido. Del contraste entre las expectativas y la percepción, el cliente llegaría al juicio de la calidad del servicio (Horovitz, 1992). Las diferentes necesidades, gustos y preferencias de los clientes, hacen de este concepto algo dinámico, expuesto a cambios. Por tanto, la calidad no está definida por la organización, sino por los propios clientes (Grönroos, 1994).

El significado de calidad ha evolucionado a lo largo de los años, desde el énfasis marcado en el control y las técnicas de inspección, pasando por la posterior atención en el concepto de aseguramiento para garantizar un nivel continuo de excelencia; hasta llegar al concepto actual de calidad total. Este último, se podría decir que es el más evolucionado y el que se acerca 
Blázquez, A.; Feu, S. (2010). Sistema de codificación para el análisis de los indicadores de calidad de las cartas de servicios en materia deportiva. Revista Internacional de Ciencias del Deporte. 19(6), 112-127. http://www.cafyd.com/REVISTA/01902.pdf

más a la idea de mejora continua. La calidad total persigue la satisfacción tanto del cliente interno como del externo, así como de los medios que se utilizan para lograrlo.

Los Sistemas de Calidad son herramientas que se centran en garantizar que lo que ofrece una entidad cumpla unas especificidades establecidas previamente por dicha entidad y el cliente. Las más conocidas son las Normas ISO y las EFQM. Otras técnicas avanzadas de gestión de la calidad son los círculos de comparación o benchmarking (Boxwell, 1994), y la reingeniería de procesos (Escobar y González, 2007).

En la gestión de la calidad en los servicios deportivos un aspecto fundamental es conocer la satisfacción de los usuarios. Entre los instrumentos más utilizados está SERVQUAL, Service Quality. Sus autores (Zeithaml, Parasuraman y Berry, 1993), establecieron los diez determinantes de la calidad de servicio. Para ello, utilizaron una escala de 22 ítems acerca de las expectativas de los usuarios del servicio, agrupados en 5 dimensiones: tangibles, empatía, fiabilidad, capacidad de respuesta y seguridad. Sin embargo, a pesar de ser la más utilizada, también es criticada por la inclusión de expectativas en las escalas o la estabilidad de las dimensiones en diferentes tipos de servicios (Babakus y Boller, 1992; Brady, Cronin, y Brand, 2002; Carman, 1990; Cronin y Taylor, 1992; Teas, 1993).

Algunos trabajos sobre el estudio de la calidad en las entidades locales y supralocales se han centrado en los programas de actividad física y deportiva basándose en la escala SERVQUAL (Dorado y Gallardo, 2003; Hernández, 2001; Mañas, Giménez, Muyor, Martínez y Moliner, 2008; Morales, Hernández y Blanco, 2005; Palacios, Fulgueisas y Catalina, 2002). Palacios, et al., (2002) diseñaron un instrumento para la medición de la calidad de los Servicios Socioculturales de Municipales, inspirándose en el modelo EFQM y en los indicadores de la escala SERVQUAL, organizándolo en tres grandes dimensiones: instalaciones, profesionales y trámites administrativos.

Dorado y Gallardo (2003) determinaron una serie de indicadores de los servicios deportivos municipales, teniendo como criterios generales los siguientes: la realidad de los servicios deportivos, los agentes que lo intervienen y el estudio de los sistemas de medición y análisis de la calidad percibida. Estos autores definen los siguientes factores en la prestación de servicios deportivos a partir de las expectativas de los usuarios: factores económicos, humanos, materiales, instalaciones deportivas, funcionamiento general, calidad global y por último quejas y sugerencias.

En la gestión de los servicios deportivos, Gallardo y Jiménez (2004) introducen los parámetros de eficacia y eficiencia. Para lograrlos, proponen que el sistema de calidad en una entidad local se apoye en cinco herramientas básicas: el manual de calidad y procedimiento del servicio deportivo, la creación de un sistema de participación y mejora de procesos, la elaboración de las cartas de servicios, la realización de estudios de valoración de la calidad del servicio y la definición de un sistema de atención de las reclamaciones y sugerencias de los clientes, con quejas, sugerencias y opiniones sobre el servicio deportivo local.

\section{Las cartas de servicio como documento de calidad}

Dentro de los documentos para la gestión de la calidad en una entidad deportiva se encuentra la carta de servicio, la cual detalla los niveles de calidad que la ciudadanía puede esperar de dichas entidades haciendo explicita la responsabilidad de los gestores públicos y desarrolla un nivel de concreción importante plasmando compromisos, indicadores, métodos de medida, etc. (Junta de Andalucía, 2004). Estos documentos tienen su origen en 1991 en el Reino 
Blázquez, A.; Feu, S. (2010). Sistema de codificación para el análisis de los indicadores de calidad de las cartas de servicios en materia deportiva. Revista Internacional de Ciencias del Deporte. 19(6), 112-127. http://www.cafyd.com/REVISTA/01902.pdf

Unido, extendiéndose a partir de aquí por la mayoría de los países europeos hasta llegar a España en 1999.

El Real Decreto 951/2005, de 29 de julio, por el que se establece el marco general para la mejora de la calidad en la Administración General de Estado presenta las Cartas de Servicios como un elemento esencial para responder a las necesidades de la ciudadanía incluyéndose también la estructura de contenido de las mismas: información de carácter general y legal, compromisos de calidad y medidas de subsanación e información de carácter complementario.

Para su elaboración, es necesario realizar un buen diagnóstico de la entidad detectando sus fines, la normativa de aplicación y definir lo más posible los servicios que ofrecen a la ciudadanía. Generalmente se diseña la carta interna, donde se recogen los procedimientos de control y seguimiento de los compromisos adquiridos; la carta pública, donde se recoge de forma detallada los servicios y compromisos de calidad adquiridos, y el folleto divulgativo, donde se resumen los indicadores y compromisos de dichos servicios.

En el diseño de los compromisos de calidad es importante comenzar por la detección de los puntos clave o centros de interés sobre los que se quiere basar la calidad. Seguidamente, se determinan los indicadores o criterios objetivos sobre los que medir dicho punto clave; para finalmente, determinar las metas o estándares que queremos alcanzar definiendo así el compromiso (Junta de Andalucía, 2004).

Para la evaluación de la gestión deportiva se hace necesario articular instrumentos que permitan su análisis (González, 2004). En la revisión realizada no se han encontrado instrumentos que permitan evaluar y analizar las cartas de servicio. El objetivo de este trabajo es crear un sistema de categorías que permita analizar los indicadores que componen los compromisos de calidad de las cartas de servicio. El análisis de los indicadores permitirá describir los elementos y temáticas a los que se dirigen los compromisos de calidad y comprobar el centro de interés de los gestores a la hora de redactar la carta de servicios. Esta información podrá contrastarse en estudios posteriores con la encuestas de satisfacción de los clientes.

\section{Método}

Desde un enfoque inductivo a partir de datos secundarios procedentes de las cartas de servicios y a partir de la deducción de las aproximaciones que otros estudios han realizado sobre la calidad en la gestión de los servicios deportivos, se ha elaborado y validado un instrumento que permite el análisis de los indicadores de calidad. Para su realización se utilizó una metodología cualitativa que utilizaba el análisis de contenido de los indicadores de calidad. En la organización del proceso de análisis de contenidos se siguieron las fases propuestas por Moreno, Santos, Ramos, Sanz, Fuentes y del Villar (2002): Organización del análisis, Categorización, Proceso de entrenamiento de codificadores y Proceso de codificación.

\section{Muestra}

Inicialmente se realizó una búsqueda a través de las páginas web de las Comunidades Autónomas, Mancomunidades y Ayuntamientos para localizar cartas de servicio que fueran públicas. Se analizaron los compromisos de calidad en el ámbito deportivo de entidades públicas de distinto nivel, Mancomunidades de Extremadura y Ayuntamiento de Madrid. La calidad de dato de la codificación se evaluó con 30 indicadores, siendo esta cifra superior al 
Blázquez, A.; Feu, S. (2010). Sistema de codificación para el análisis de los indicadores de calidad de las cartas de servicios en materia deportiva. Revista Internacional de Ciencias del Deporte. 19(6), 112-127. http://www.cafyd.com/REVISTA/01902.pdf

número de indicadores que suele tener una carta de servicios destinada al ámbito deportivo o a un área del mismo.

\section{Categorización}

Inicialmente se establecieron tres dimensiones a las que le correspondían una serie de categorías generales: dimensión Objeto (7), dimensión Temática (7) y dimensión Temporalidad (5). Tras la primera sesión y codificación se decidió ampliar la categorización para mejorar la validez de contenido y responder al consenso interobservadores, estableciendo las siguientes categorías generales: dimensión Objeto (9), dimensión Temática (11) y dimensión Temporalidad (6).

\section{Dimensión 1: Objeto}

Esta dimensión describe los elementos medibles (tangibles o intangibles), que componen el objeto directo del enunciado del compromiso calidad y hacia dónde directamente se dirige la acción a realizar. Se trata de una dimensión donde sus categorías son excluyentes entre sí, de ahí la importancia de seleccionar el núcleo del objeto directo. Haría referencia sobre qué o quién se dirige la medida. En esta dimensión se incluyen nueve categorías (Tabla 2):

1) Procedimientos: son todos los compromisos que hacen referencia a los procesos, entendiendo por tales aquellas acciones encaminadas a facilitar la consecución de un fin (Dimensión Temática). Se pueden subclasificar según la orientación de la acción (Heras, 1996):

o Procesos estratégicos: aquellos encaminados a proporcionar directrices a todos los demás procesos; suelen estar más relacionados con la dirección o por otras entidades (p.e.: colaboraciones con otras entidades, compras de instalaciones o material de gran coste).

o Procesos clave: atañen a diferentes áreas del Servicio y tienen impacto en el cliente creando valor para éste. En el caso de los servicios deportivos serían aquellas acciones relacionadas con la actividad físico-deportiva. Por ejemplo: metodología de enseñanza, tiempos de duración de una clase...

o Procesos de soporte: Son los procesos que ayudan a la hora de realizar nuestros procesos fundamentales. Ejemplo: atención telefónica, limpieza de una instalación,...

2) Actividades: son los compromisos que se refieren a actividades que desarrolla el servicio deportivo. Las actividades según su duración pueden ser: Puntuales, con una corta duración, inferior a un mes y desarrollada en un momento concreto de la planificación anual (p.e. jornadas, campañas, convivencias, torneos, campeonatos etc.); Periódicas, desarrollada a lo largo de una temporada con una continuidad superior a un mes (p.e. programas, escuelas deportivas, etc.); No definida, donde incluiríamos todas aquellas que generen dudas a la hora de incluirla en los subapartados anteriores.

3) Usuarios (cliente externo): son los participantes o clientes de los servicios deportivos y grupos de personas no pertenecientes a una entidad legalizada. Incluiríamos aquí aquellos casos donde para la participación puntual en una actividad obliga a hacerlo bajo el nombre de un colectivo. Por tanto, las dos subcategorías más importantes son individual y grupo-equipo.

4) Entidades (cliente externo): son los colectivos legalmente constituidos. Las subcategorías que podemos encontrar son: Empresas (autónomos y sociedades), 
Blázquez, A.; Feu, S. (2010). Sistema de codificación para el análisis de los indicadores de calidad de las cartas de servicios en materia deportiva. Revista Internacional de Ciencias del Deporte. 19(6), 112-127. http://www.cafyd.com/REVISTA/01902.pdf

Entidades sin ánimo de lucro (asociaciones, agrupaciones, fundaciones) y Administraciones públicas (ámbito local, regional, nacional o internacional).

5) Recursos humanos (cliente interno): constituidos por aquellas personas vinculadas directamente o indirectamente a la entidad y que desarrollan o ayudan a desarrollar la actividad de la misma. Son los propios trabajadores asalariados de la entidad que desarrollan compromisos relacionados con el servicio deportivo y los colaboradores o voluntarios.

6) Documentos: el Instituto Nacional de Seguridad e Higiene en el Trabajo (2007) los define como el soporte de cierta duración temporal en el que se haya registrado cualquier conocimiento o experiencia humana. Según el tipo de registro se pueden diferenciar: Soporte gráfico (fotografías, esquemas, libros,...), Sonoros (cinta magnética, discos,...), Audiovisuales (película, montaje audivisual,..), Digital (páginas web, aplicaciones informáticas...) o Táctiles (escritura Braille).

7) Instalaciones: son los espacios para la práctica deportiva y espacios complementarios. El Censo Nacional de Instalaciones Deportivas clasifica los espacios como (Consejo Superior de Deportes, 2005): Espacios convencionales, Áreas de actividad, Espacios singulares y Espacios complementarios.

8) Recursos materiales: Esta categoría se centra en el concepto de equipamiento y material deportivo. Clasificando el material en función de su duración este puede ser: inventariable o de una duración o consumo mayor de un año. (p.e.: canastas, colchonetas) y Fungible o de una duración o consumo inferior a un año (p.e.: balones, redes, etc.).

9) Recursos económicos: esta categoría se refiere a aquellos activos que hacen referencia al término transacción de común acuerdo entre las partes (p.e. premios, subvención, etc.). La subclasificación propuesta es la siguiente:

o Dinero fiduciario: se refiere a los billetes y monedas como los elementos de intercambio legal, y al concepto de presupuesto de una entidad, cuantificado en una cantidad económica.

o Dinero bancario: se trata de un medio de cambio basado en la deuda de una institución de crédito, también acordados legalmente. Aquí se incluyen los cheques, transferencias, subvenciones, etc.

o Dinero trueque, donde se recogen aquellos casos de intercambios de pagos en especie y que son válidos para las dos partes que intervienen, pero que no tienen por qué ser válidos en otros lugares. Ejemplos: entradas, bonos, vales, premios, trofeos, etc. Aquí se incluye el concepto de precio de los documentos o materiales anteriores.

\section{Dimensión 2: Temática.}

Esta dimensión describe el asunto al que se refiere la acción que se quiere llevar a cabo a través del compromiso y/o indicadores que lo definen. Haría referencia al "para qué” se dirige la acción y que definiría la temática del objeto. En algunos casos una sola categoría define al objeto directo del indicador y en otros casos pueden ser varias. El desglose de las once categorías de esta dimensión se ha distribuido en áreas de gestión (Tabla 1). 
Blázquez, A.; Feu, S. (2010). Sistema de codificación para el análisis de los indicadores de calidad de las cartas de servicios en materia deportiva. Revista Internacional de Ciencias del Deporte. 19(6), 112-127. http://www.cafyd.com/REVISTA/01902.pdf

Tabla 1.- Subclasificación de las categorías pertenecientes a la dimensión Temática.

\begin{tabular}{|l|l|}
\hline \multicolumn{1}{|c|}{ Área } & \multicolumn{1}{c|}{ Categoría temática } \\
\hline Gestión de la productividad & $\begin{array}{l}\text { Tamaño (cuantifica, aumenta, disminuye, crea o elimina) } \\
\text { Rendimiento (compara, clasifica, ordena) }\end{array}$ \\
\hline $\begin{array}{l}\text { Gestión de las cualidades de seguridad } \\
\text { y confort }\end{array}$ & $\begin{array}{l}\text { Seguridad (previene) } \\
\text { Condiciones ambientales (garantiza confort) }\end{array}$ \\
\hline Gestión de la relación entre personas & $\begin{array}{l}\text { Igualdad de oportunidades (relación entre sexos) } \\
\text { Intergeneracional (relación entre diferentes edades) } \\
\text { Accesibilidad-inclusión (facilita el acceso a personas con con } \\
\text { discapacidad física, psíquica, sensorial o social) }\end{array}$ \\
\hline Gestión del know how & Función general (cómo se hace) \\
\hline $\begin{array}{l}\text { Gestión del conocimiento y la } \\
\text { información }\end{array}$ & $\begin{array}{l}\text { Marketing (informa hacia el exterior) } \\
\text { Retroalimentación (conoce información del exterior) } \\
\text { Formación (asimila información) }\end{array}$ \\
\hline
\end{tabular}

1) Tamaño: se trata de una categoría referida a los aspectos cuantitativos del objeto y donde se recogen todas aquellas medidas encaminadas a crear, eliminar, modificar o mantener la cuantía del objeto en sí mismo y no con ninguna otra finalidad adicional que viniese expresamente reflejada.

2) Rendimiento: este apartado engloba el concepto de productividad, comparación, clasificación o tecnificación del objeto al que hace referencia. Esta categoría se diferencia de la de Tamaño en que para su consecución debe compararse con otros valores.

3) Marketing: son aquellos referidos a la imagen, comunicación externa de la entidad, etc., asociadas al concepto de difusión y referidas a las categorías de la dimensión objeto. Recogeríamos aquí la publicidad, campañas de información, etc. El sentido de la información es desde dentro hacia fuera de la entidad.

4) Retroalimentación: hace referencia a todos los sistemas de información y comunicación que pretenden evaluar y dotar de mayor conocimiento de la gestión de la entidad y de los propios procedimientos implantados. Aquí se incluiría todos aquellos sistemas de valoración de la satisfacción, sugerencias, reclamaciones etc.; de usuarios, personal propio y entidades. Se diferenciaría de la categoría marketing en el sentido de la comunicación, que en este caso sería de fuera hacia dentro.

5) Formación: son todas las actuaciones encaminadas a la enseñanza - aprendizaje de los sujetos a los que se dirige la acción (cursos, seminarios, jornadas,...).

6) Condiciones ambientales: aquellas acciones relacionadas con los aspectos cualitativos de la dimensión objeto y asociadas al concepto de confort. Estos aspectos generalmente se refieren a las condiciones ambientales y contextuales del servicio deportivo: limpieza, sonorización, iluminación, temperatura, humedad, decoración,...

7) Seguridad: son aquellas acciones que se relacionan con la seguridad de las categorías señaladas en la dimensión objeto.

8) Accesibilidad-inclusión: se refiere al concepto de inclusión como aquellas medidas que se dirigen a facilitar el acceso de personas con algún tipo de discapacidad (física, psíquica y sensorial) o exclusión social en relación a las categorías señaladas en la dimensión objeto.

9) Igualdad de oportunidades: se refiere al concepto de coeducación y de igualdad de oportunidades entre ambos sexos.

10) Intergeneracional: se refiere a los indicadores cuya temática trata sobre la relación entre personas de diferente edad entre sí, dentro de una misma actividad, proceso, etc.

11) Funcionamiento general: esta categoría recoge aquellos aspectos relacionados con el funcionamiento, modo de llevarlo a cabo o know how (saber hacer) de la categoría 
Blázquez, A.; Feu, S. (2010). Sistema de codificación para el análisis de los indicadores de calidad de las cartas de servicios en materia deportiva. Revista Internacional de Ciencias del Deporte. 19(6), 112-127. http://www.cafyd.com/REVISTA/01902.pdf

señalada en la dimensión objeto y que no hacen referencia a las categorías anteriores. Así algunas temáticas relacionadas son: metodología (horarios, metodologías de intervención, etc.), calidad de la atención (amabilidad, etc.), normalización (reglamentos, normativas, etc.), innovación (tecnología, procesos, etc.); así como todos aquellos aspectos relacionados con la orientación estratégica de la entidad (acuerdos, colaboraciones...).

\section{Dimensión 3: Temporal}

Esta dimensión describe el tiempo de medida en que se evaluará el indicador. Hace referencia a cuándo se medirá el compromiso. Las cartas de calidad suelen medir sus indicadores a lo largo de un año. Se pueden considerar varios momentos temporales excluyentes entre sí: Fin Acción, Mensual, Bimensual, Trimestral, Semestral y Anual (Tabla 2).

Tabla 2.- Categorización de los indicadores de compromisos de calidad de las Cartas de Servicio.

\begin{tabular}{|c|c|c|c|}
\hline Dimensión & Categoría & Código & Descripción \\
\hline \multirow[t]{9}{*}{ Objeto } & Usuario & USUA & $\begin{array}{l}\text { Participantes o clientes de los servicios deportivos y grupos de personas } \\
\text { no pertenecientes a una entidad legalizada. }\end{array}$ \\
\hline & Entidad & ENTI & Colectivos legalmente constituidos. \\
\hline & $\begin{array}{l}\text { Recursos } \\
\text { humanos }\end{array}$ & RRHH & $\begin{array}{l}\text { Personas vinculadas directamente o indirectamente a la entidad y que } \\
\text { desarrollan o ayudan a desarrollar la actividad de la misma. }\end{array}$ \\
\hline & Documentos & DOC & $\begin{array}{l}\text { Soporte de cierta duración temporal en el que se registra cualquier } \\
\text { conocimiento o experiencia humana. }\end{array}$ \\
\hline & Instalaciones & INSTA & Espacios deportivos y complementarios. \\
\hline & \begin{tabular}{|l|} 
Recursos \\
Materiales \\
\end{tabular} & RRMA & Equipamiento o material deportivo, inventariable o fungible. \\
\hline & $\begin{array}{l}\text { Recursos } \\
\text { Económicos }\end{array}$ & RREC & $\begin{array}{l}\text { Activos que hacen referencia al término transacción de común acuerdo } \\
\text { entre las partes. }\end{array}$ \\
\hline & Actividades & ACTI & Actividades que desarrolla el servicio deportivo. \\
\hline & Procedimientos & PROC & $\begin{array}{l}\text { Hace referencia a los procesos, a aquellas acciones encaminadas a } \\
\text { facilitar la consecución de un fin. }\end{array}$ \\
\hline \multirow[t]{11}{*}{ Temática } & Tamaño & TAM & $\begin{array}{l}\text { Aspectos cuantitativos del objeto y donde se recogen todas aquellas } \\
\text { medidas encaminadas a crear, eliminar, modificar o mantener la cuantía } \\
\text { del objeto en sí mismo. }\end{array}$ \\
\hline & Rendimiento & REND & $\begin{array}{l}\text { Productividad, comparación, clasificación o tecnificación del objeto al } \\
\text { que hace referencia. }\end{array}$ \\
\hline & $\begin{array}{l}\text { Condición } \\
\text { ambiental }\end{array}$ & AMBI & $\begin{array}{l}\text { Acciones asociadas al concepto de confort. Condiciones ambientales y } \\
\text { contextuales del servicio deportivo: limpieza, sonorización, iluminación, } \\
\text { temperatura, humedad, decoración,... }\end{array}$ \\
\hline & Intergeneracional & INTER & $\begin{array}{l}\text { Acciones encaminadas a favorecer la relación entre personas de } \\
\text { diferente edad entre sí. }\end{array}$ \\
\hline & $\begin{array}{l}\text { Igualdad de } \\
\text { oportunidades }\end{array}$ & IGUA & $\begin{array}{l}\text { Acciones encaminadas a favorecer la coeducación y de } \rightarrow \text { la igualdad de } \\
\text { oportunidades entre ambos sexos. }\end{array}$ \\
\hline & Seguridad & SEGU & $\begin{array}{l}\text { Acciones relacionadas con la seguridad de las categorías señaladas en } \\
\text { la dimensión objeto. }\end{array}$ \\
\hline & $\begin{array}{l}\text { Accesibilidad - } \\
\text { Inclusión }\end{array}$ & ACCE & $\begin{array}{l}\text { Medidas que se dirigen a facilitar el acceso de personas con algún tipo } \\
\text { de discapacidad o exclusión social en relación a las categorías. }\end{array}$ \\
\hline & Marketing & MARK & $\begin{array}{l}\text { Aspectos referidos a la imagen, comunicación externa de la entidad, } \\
\text { etc., asociadas al concepto de difusión y referidas a las categorías de la } \\
\text { dimensión objeto. }\end{array}$ \\
\hline & Formación & FORM & $\begin{array}{l}\text { Acciones encaminadas a la formación (cursos, acciones formativas, } \\
\text { jornadas...) }\end{array}$ \\
\hline & Retroalimentación & RETRO & $\begin{array}{l}\text { Sistemas de información y comunicación para evaluar y dotar de } \\
\text { mayor conocimiento de la gestión de la entidad y de los propios } \\
\text { procedimientos implantados. }\end{array}$ \\
\hline & Función General & FGEN & $\begin{array}{l}\text { Aspectos relacionados con el funcionamiento y el modo de llevar a cabo } \\
\text { (saber hacer) la categoría señalada en la dimensión objeto y que no } \\
\text { hacen referencia a las categorías anteriores de la dimensión temática. }\end{array}$ \\
\hline
\end{tabular}


Blázquez, A.; Feu, S. (2010). Sistema de codificación para el análisis de los indicadores de calidad de las cartas de servicios en materia deportiva. Revista Internacional de Ciencias del Deporte. 19(6), 112-127. http://www.cafyd.com/REVISTA/01902.pdf

\begin{tabular}{|l|l|l|l|}
\hline Temporalidad & Fin de la acción & FIN & Medición del indicador cuando finaliza la propia acción. \\
\cline { 2 - 4 } & Mensual & MES & Medición del indicador con periodicidad desde 1 día hasta un mes. \\
\cline { 2 - 4 } & Bimensual & BMES & $\begin{array}{l}\text { Medición del indicador con periodicidad a partir de un mes hasta antes } \\
\text { de tres meses. }\end{array}$ \\
\cline { 2 - 4 } & Trimestral & TMES & $\begin{array}{l}\text { Medición del indicador con periodicidad desde } 3 \text { meses hasta antes de } \\
\text { seis meses. }\end{array}$ \\
\cline { 2 - 5 } & Semestral & SMES & $\begin{array}{l}\text { Medición del indicador con periodicidad desde } 6 \text { meses hasta antes de } \\
\text { un año. }\end{array}$ \\
\cline { 2 - 5 } & Anual & AÑO & Medición del indicador con periodicidad de un año. \\
\hline
\end{tabular}

\section{Procedimiento de codificación y entrenamiento de observadores}

Para determinar la validez y fiabilidad del instrumento fue necesario contar con tres codificadores. Los codificadores eran Licenciados en Ciencias de la Actividad Física y el Deporte que trabajan en la administración pública. El procedimiento de entrenamiento de los codificadores se desarrolló en tres fases (Delgado y del Villar, 1994).

Primera fase: se estableció la unidad de contenido que se iba analizar, decidiéndose utilizar el párrafo que describe cada indicador de calidad y que a su vez, define total o parcialmente el compromiso de calidad.

Segunda fase: a partir de un proceso deductivo-inductivo (Medina, 1996), se analizaron las investigaciones que afrontaron objetivos similares y se delimitaron los objetivos del estudio y el alcance en función de las características del material a analizar, los compromisos de calidad de las cartas de servicio de las treinta Mancomunidades de Extremadura y del Ayuntamiento de Madrid. Se estudiaron todos los compromisos de calidad de las cartas de servicio seleccionadas decidiendo finalmente analizar 40 indicadores extraídos al azar.

Seguidamente se establecieron tres grandes dimensiones o categorías y se desglosaron en diferentes subcategorías, asociando cada una de ellas a una abreviatura. Se explicaron las categorías y subcategorías iniciales a los codificadores, mostrando algunos ejemplos. Se realizaron cuatro sesiones de valoración en días diferentes valorando diez compromisos de calidad en cada una de ellas, tardándose en cada análisis una media de treinta minutos. Antes de cada prueba se procedía a resolver las dudas y se debatían las diferencias encontradas y las aportaciones de los codificadores con el objetivo de alcanzar una concordancia consensuada (Anguera, 1990; Castellano, Hernández, Gómez de Segura, Fontexa y Bueno, 2000). La validez de contenido de cada definición se discutió en el equipo de investigación y con los codificadores.

Teniendo en cuenta que un indicador de calidad puede escribirse en forma de una oración gramatical simple, es posible el análisis sintáctico de los elementos de la misma para poder categorizar la información que ofrecen. El codificador puede diferenciar el sujeto (entidad que emite el indicador), el objeto directo (elemento sobre el que recae la acción del verbo), el verbo (diferenciado la temática de su acción) o el complemento circunstancial que expresa el momento de medida del indicador. No suele ser frecuente la aparición de complementos indirectos. El proceso de codificación de los indicadores en este estudio se realiza en base al análisis de tres elementos: objeto directo, temática de la acción del verbo y complemento circunstancial de tiempo de medición del mismo.

La dimensión temática agrupa categorías no excluyentes entre sí, a excepción de la categoría Tamaño y Función general. La categoría Tamaño permite codificar la temática del indicador cuando no queda claramente expresamente indicada por otra categoría específica de esta 
Blázquez, A.; Feu, S. (2010). Sistema de codificación para el análisis de los indicadores de calidad de las cartas de servicios en materia deportiva. Revista Internacional de Ciencias del Deporte. 19(6), 112-127. http://www.cafyd.com/REVISTA/01902.pdf

dimensión; mientras que la categoría Función General tiene la función de englobar todas aquellas temáticas relacionadas con el saber hacer y donde se incluirían matices desde la amabilidad en la atención, hasta normalizar o mejorar los tiempos en los procedimientos, cambios en la metodología de enseñanza...

Tercera fase: se calculó el grado de acuerdo intercodificadores e intracodificador principal, tomados dos a dos (Krippendorff, 1990), y un análisis cuantitativo de los acuerdos intercodificador e intracodificador (Gorospe, Hernández, Anguera, y Martínez de Santos, 2005).

\section{Análisis estadístico}

Para determinar la calidad de dato del instrumento desarrollado para la codificación de los indicadores de los compromisos de calidad, se analizaron los índices de acuerdo y de asociación inter e intra observador. A través del Coeficiente de Correlación Intraclase se calculó el índice de acuerdo entre los observadores para discriminar las frecuencias de todas las categorías (García de Yébenes, Rodríguez y Carmona, 2009). Mediante el índice Kappa de Cohen se analizó la asociación entre las observaciones, tomadas dos a dos, teniendo en cuenta los errores por efecto del azar y el orden en el que los codificadores han registrado las categorías (García de Yébenes, et al., 2009; Gorospe, et al., 2005). Para los análisis se utilizó el software para análisis estadístico Excel y SPSS 15.

\section{Resultados}

Inicialmente se analizó la distribución de las frecuencias obtenidas en cada categoría en total y por sesión (Tabla 3). Los resultados indican que los compromisos de calidad analizados han discriminado dieciséis de las veinte variables previstas, no registrando una variable en la dimensión Objeto, los Recursos humanos, y no registrando tres tipos de temática: Intergeneracional, Igualdad de oportunidades y Seguridad. Los resultados indican un porcentaje de acuerdo interobservador tomados dos a dos con el observador 3 (observador principal) superior al 90\%. La media del porcentaje de acuerdo del observador 1 y 2 con el observador 3 es mayor o igual a .95. 
Blázquez, A.; Feu, S. (2010). Sistema de codificación para el análisis de los indicadores de calidad de las cartas de servicios en materia deportiva. Revista Internacional de Ciencias del Deporte. 19(6), $112-127$. http://www.cafyd.com/REVISTA/01902.pdf

Tabla 3. Frecuencias de las observaciones y porcentaje de acuerdo inter e intra observador.

\begin{tabular}{|c|c|c|c|c|c|c|c|c|c|c|c|c|c|c|c|c|c|}
\hline & \multicolumn{13}{|c|}{ Frecuencias } & \multicolumn{4}{|c|}{$\begin{array}{c}\begin{array}{c}\% \text { de acuerdo del total de } \\
\text { observaciones }\end{array} \\
\end{array}$} \\
\hline & \multicolumn{3}{|c|}{ Sesión 1} & \multicolumn{3}{|c|}{ Sesión 2} & \multicolumn{3}{|c|}{ Sesión 3} & \multicolumn{4}{|c|}{ Total } & \multicolumn{4}{|c|}{\begin{tabular}{|l|l|} 
intraobservador & interobservador
\end{tabular}} \\
\hline & Obs1 & Obs2 & Obs3 & Obs1 & Obs2 & Obs3 & Obs1 & Obs2 & Obs3 & Obs1 & Obs2 & Obs3 & $\begin{array}{c}\text { Obs3 } \\
\text { (bis) }\end{array}$ & $\begin{array}{l}\text { Obs3 \& Obs3 } \\
\text { (bis) }\end{array}$ & $\begin{array}{c}\text { Obs1 } \\
\& \\
\text { Obs3 }\end{array}$ & $\begin{array}{c}\text { Obs2 } \\
\& \\
\text { Obs3 }\end{array}$ & $M$ \\
\hline USUA & 2 & 2 & 2 & 5 & 5 & 5 & 0 & 0 & 0 & 7 & 7 & 7 & 7 & 100 & 100 & 100 & 100.00 \\
\hline ENTI & 2 & 2 & 2 & 1 & 1 & 1 & 0 & 0 & 0 & 3 & 3 & 3 & 3 & 100 & 100 & 100 & 100.00 \\
\hline RRHH & 0 & 0 & 0 & 0 & 0 & 0 & 0 & 0 & 0 & 0 & 0 & 0 & 0 & 100 & 100 & 100 & 100.00 \\
\hline DOC & 1 & 1 & 1 & 2 & 1 & 1 & 1 & 1 & 1 & 4 & 3 & 3 & 3 & 96.7 & 96.7 & 100 & 98.35 \\
\hline INSTA & 0 & 0 & 0 & 0 & 0 & 0 & 4 & 3 & 3 & 4 & 3 & 3 & 3 & 96.7 & 96.7 & 100 & 98.35 \\
\hline RRMA & 0 & 0 & 0 & 0 & 0 & 0 & 1 & 1 & 1 & 1 & 1 & 1 & 1 & 100 & 100 & 100 & 100.00 \\
\hline RREC & 0 & 0 & 0 & 0 & 0 & 0 & 1 & 0 & 1 & 1 & 0 & 1 & 1 & 100 & 100 & 96.7 & \begin{tabular}{|r|}
98.35 \\
\end{tabular} \\
\hline ACTI & 5 & 5 & 5 & 2 & 2 & 2 & 3 & 4 & 4 & 10 & 11 & 11 & 11 & 100 & 100 & 96.7 & 98.35 \\
\hline PROC & 0 & 0 & 0 & 1 & 1 & 1 & 1 & 0 & 0 & 2 & 1 & 1 & 1 & 100 & 100 & 100 & 100.00 \\
\hline TAM & 5 & 3 & 4 & 6 & 6 & 6 & 2 & 2 & 2 & 13 & 11 & 12 & 13 & 90 & 96.7 & 96.7 & 96.70 \\
\hline REND & 1 & 2 & 2 & 0 & 0 & 0 & 2 & 1 & 2 & 3 & 3 & 4 & 2 & 93.3 & 96.7 & 96.7 & \begin{tabular}{|l|}
96.70 \\
\end{tabular} \\
\hline AMBI & 0 & 0 & 0 & 0 & 0 & 0 & 1 & 1 & 1 & 1 & 1 & 1 & 2 & 96.7 & 100 & 100 & 100.00 \\
\hline INTER & 0 & 0 & 0 & 0 & 0 & 0 & 0 & 0 & 0 & 0 & 0 & 0 & 0 & 100 & 100 & 100 & 100.00 \\
\hline IGUA & 0 & 0 & 0 & 0 & 0 & 0 & 0 & 0 & 0 & 0 & 0 & 0 & 0 & 100 & 100 & 100 & 100.00 \\
\hline SEGU & 0 & 0 & 0 & 0 & 0 & 0 & 0 & 0 & 0 & 0 & 0 & 0 & 0 & 100 & 100 & 100 & 100.00 \\
\hline ACCE & 0 & 0 & 0 & 1 & 1 & 1 & 0 & 1 & 0 & 1 & 2 & 1 & 1 & 100 & 100 & 96.7 & 98.35 \\
\hline MARK & 1 & 1 & 1 & 1 & 1 & 1 & 1 & 1 & 1 & 3 & 3 & 3 & 2 & 96.7 & 100 & 100 & 100.00 \\
\hline FORM & 0 & 0 & 0 & 0 & 0 & 0 & 1 & 1 & 1 & 1 & 1 & 1 & 1 & 100 & 100 & 100 & 100.00 \\
\hline RETRO & 1 & 1 & 1 & 1 & 1 & 1 & 1 & 1 & 1 & 3 & 3 & 3 & 3 & 100 & 100 & 100 & 100.00 \\
\hline FGEN & 2 & 2 & 2 & 1 & 1 & 1 & 2 & 2 & 2 & 5 & 5 & 5 & 5 & 93.3 & 100 & 93.3 & \begin{tabular}{|l}
96.65 \\
\end{tabular} \\
\hline FIN & 0 & 0 & 0 & 1 & 1 & 1 & 0 & 0 & 0 & 1 & 1 & 1 & 1 & 100 & 100 & 100 & 100 \\
\hline MES & 0 & 0 & 0 & 3 & 3 & 3 & 0 & 0 & 0 & 3 & 3 & 3 & 3 & 100 & 100 & 100 & 100 \\
\hline BMES & 0 & 0 & 0 & 1 & 1 & 1 & 0 & 0 & 0 & 1 & 1 & 1 & 1 & 100 & 100 & 100 & 100 \\
\hline TMES & 1 & 1 & 1 & 1 & 1 & 1 & 0 & 0 & 0 & 2 & 2 & 2 & 2 & 100 & 100 & 100 & 100 \\
\hline SMES & 0 & 0 & 0 & 0 & 0 & 0 & 0 & 0 & 0 & 0 & 0 & 0 & 0 & 100 & 100 & 100 & 100 \\
\hline AÑO & 9 & 9 & 9 & 4 & 4 & 4 & 10 & 10 & 10 & 23 & 23 & 23 & 23 & 100 & 100 & 100 & 100 \\
\hline
\end{tabular}

Desde una perspectiva cuantitativa se estudió la concordancia consensuada interobservador para discriminar las frecuencias de todas las categorías objeto de estudio. Los resultados arrojados por el coeficiente de correlación intraclase, con intervalo de confianza al 95\%, indican que hay una alta fiabilidad entre los observadores (CCI = .948; IC: 990-998; p<.01). Se realizó el mismo procedimiento con las frecuencias obtenidas en dos momentos diferentes por el observador principal obteniéndose un coeficiente de correlación intraclase que indicaba un alto consenso entre ambas observaciones (CCI =.995; IC: 988-998; $\mathrm{p}<.01$ ).

A través del índice de kappa se analizó la existencia de una asociación entre las observaciones contrastadas teniendo en cuenta los errores por efecto del azar y el orden en el que los codificadores han registrado las categorías. Los resultados indican una asociación significativa $(\mathrm{p}<.01)$ más alta que la esperada por el azar en la comparación interobservadores e intraobservador principal, (Tabla 4); la concordancia es buena en algunas observaciones (valor de $\mathrm{k}>$.61) y muy buena en la mayoría de observaciones contrastradas (valor de $\mathrm{k}>.81$ ). En algunas variables, como Recursos humanos, Intergeneracional, Igualdad de oportunidades, Seguridad y temporalidad Semestral, no se pudo calcular el debido a que la serie de datos era constante debido a la coincidencia de ausencia de observaciones sobre esa variable. 
Blázquez, A.; Feu, S. (2010). Sistema de codificación para el análisis de los indicadores de calidad de las cartas de servicios en materia deportiva. Revista Internacional de Ciencias del Deporte. 19(6), 112-127. http://www.cafyd.com/REVISTA/01902.pdf

Tabla 4. Índice Kappa de Cohen de las observaciones intra e inter observadores.

\begin{tabular}{|c|c|c|c|c|c|c|}
\hline & \multicolumn{2}{|c|}{ Obs1 \& Obs3 } & \multicolumn{2}{|c|}{ Obs2 \& Obs3 } & \multicolumn{2}{|c|}{ Obs3 \& Obs3 bis } \\
\hline Categoría & valor $k$ & $p$ & valor $k$ & $p$ & valor $k$ & $p$ \\
\hline USUA & 1.00 & .000 & 1.00 & .00 & .600 & ,001 \\
\hline ENTI & 1.00 & .000 & 1.00 & .00 & 1.00 & .000 \\
\hline DOC & .839 & .000 & 1.00 & .000 & .839 & .000 \\
\hline INSTA & .839 & .000 & 1.00 & .000 & 1.00 & .000 \\
\hline RRMA & 1.00 & .000 & 1.00 & .000 & 1.00 & .000 \\
\hline RREC & 1.00 & .000 & - & - & 1.00 & .000 \\
\hline ACTI & .927 & .000 & 1.00 & .000 & 1.00 & .000 \\
\hline PROC & .651 & .000 & 1.00 & .000 & - & - \\
\hline TAM & .932 & .000 & .930 & .000 & .795 & .000 \\
\hline REND & .839 & .000 & .839 & .000 & .634 & .000 \\
\hline $\mathrm{AMBI}$ & 1.00 & .000 & 1.00 & .000 & .651 & .000 \\
\hline ACCE & 1.00 & .000 & .651 & .000 & 1.00 & .000 \\
\hline MARK & 1.00 & .000 & 1.00 & .000 & .783 & .000 \\
\hline FORM & 1.00 & .000 & 1.00 & .000 & 1.00 & .000 \\
\hline RETRO & 1.00 & .000 & 1.00 & .000 & 1.00 & .000 \\
\hline FGEN & 1.00 & .000 & .760 & .000 & .760 & .000 \\
\hline FIN & 1.00 & .000 & 1.00 & .000 & 1.00 & .000 \\
\hline MES & 1.00 & .000 & 1.00 & .000 & 1.00 & .000 \\
\hline BMES & 1.00 & .000 & 1.00 & .000 & 1.00 & .000 \\
\hline TMES & 1.00 & .000 & 1.00 & .000 & 1.00 & .000 \\
\hline AÑO & 1.00 & .000 & 1.00 & .000 & 1.00 & .000 \\
\hline
\end{tabular}

$(-)$ No se calcula el estadístico al ser una de las variables una constante.

\section{Discusión}

El instrumento propuesto para el análisis de los indicadores de los compromisos de calidad de las cartas de servicio ha seguido un proceso deductivo - inductivo con la finalidad de garantizar la validez de contenido de las dimensiones y categorías adoptadas. Para ello, se utilizó la concordancia consensuada que consistió en buscar, antes de iniciar cada sesión, acuerdos entre los codificadores en aquellos puntos que lo requerían (Anguera, 1990). Las dimensiones y categorías definidas en este instrumento abarcan un amplio espectro de elementos que pueden ser considerados para la mejora y evaluación de documentos de calidad como las cartas de servicio. Los resultados obtenidos avalan una validez interna para el estudio en profundidad de los indicadores de calidad de las cartas de servicio seleccionadas para este estudio.

El análisis de las frecuencias muestra que cuatro categorías no se han encontrado en el análisis de los indicadores, probablemente debido a que el modelo teórico prevé un amplio espectro de elementos posibles. No obstante, los compromisos de calidad de una carta de servicios no deberían ser demasiado amplios y sí concretados claramente a través de sus indicadores. Esto permitiría una definición más precisa de las líneas estratégicas de la entidad. El instrumento es adecuado, ya que permite evaluar las distintas dimensiones que abarca la calidad en los servicios públicos deportivos (Morales et al., 2005).

La calidad de dato del instrumento fue adecuada obteniendo una fiabilidad interobservadores e intraobservador que puede ser considerada óptima, ya que los acuerdos son superiores al 80\% (Goezt y LeCompte, 1988; Guba, 1981). Los resultados del Coeficiente de Correlación Intraclase y del índice Kappa de Cohen ponen de manifiesto una concordancia adecuada interobservador e intraobservador (García de Yébenes, et al, 2009; Gorospe, et al., 2005).

La tendencia en el estudio de la calidad en los servicios deportivos se ha centrado principalmente en el análisis de la satisfacción de los usuarios a través de instrumentos que se 
Blázquez, A.; Feu, S. (2010). Sistema de codificación para el análisis de los indicadores de calidad de las cartas de servicios en materia deportiva. Revista Internacional de Ciencias del Deporte. 19(6), 112-127. http://www.cafyd.com/REVISTA/01902.pdf

han ido adaptado a las características del propio servicio y de los usuarios (Calabuig y Saura, 1999; Kim y Kim, 1995; Mundina, y Calabuig, 1999; Palacios, et al., 2002; Rodríguez, Agudo, García, y Herrero, 2003).

En la implantación de un sistema de calidad, esta información debería utilizarse para la elaboración de manuales o documentos que den respuesta a la demanda de los usuarios, como por ejemplo las cartas de servicio. Las instituciones públicas deben adaptarse al entorno y buscar sistemas para ofrecer unos servicios más competitivos eficaces y transparentes (Carrasco, Buendía, Navarro, Valencia, y Llorente, 2006). El análisis de la realidad del servicio prestado y el plan de mejora puesto en marcha para mejorar la calidad deben ir acompañados de un compromiso con la ciudadanía para mejorar la calidad del mismo. Las cartas de servicio son herramientas imprescindibles que pueden y deben ser evaluadas.

\section{Conclusiones}

La herramienta para el análisis de contenido de los indicadores que definen los compromisos de calidad de las cartas de servicio presenta unos resultados totalmente satisfactorios en cuanto a la calidad de dato, tanto en los coeficientes de correlación como en los índices de fiabilidad.

El instrumento permite analizar y describir en profundidad los contenidos expresados en los indicadores de calidad de las cartas de servicio de las instituciones seleccionadas. Asimismo, se podrá comparar las líneas estratégicas de las distintas instituciones analizadas.

La herramienta presentada permite complementar el proceso inductivo realizado a partir de la opinión de los usuarios con un análisis del documento de calidad, obteniéndose así un feedback sobre de las estrategias llevadas a cabo por la entidad. El contraste entre la opinión y satisfacción de los usuarios con la orientación de las líneas estratégicas presentadas en las cartas de servicio aportarán una información útil sobre la adecuación de las medidas que ha expresado la entidad en su carta de servicios. Esto permitiría iniciar un nuevo proceso de reflexión sobre la adecuación de las mismas a las demandas de los usuarios.

Las categorías y dimensiones que constituyen la estructura de la misma permiten un mejor conocimiento de los elementos que conforman el sistema deportivo local.

\section{Referencias}

Anguera, M. T. (1990). Metodología observacional. En J. Arnau, M. T. Anguera, y J. Gómez. Metodología de la observación en las ciencias del comportamiento (pp. 125236) Murcia: Universidad de Murcia.

Ayuntamiento de Madrid (2008). Carta de Servicios. Obtenido el 5 de junio de 2009 desde http://www-1.munimadrid.es/CS/listaCompromisos.do

Bakakus, E.; Boller, G. W. (1992). An empirical assessment of the SERVQUAL scale. J ournal of Business Research, 24(3), 253-268.

Boxwell R. J. (1994) Benchmarking para competir con ventaja. McGraw Hill. Madrid

Brady, K. M.; Cronin, J. J., y Brand, R. R. (2002). Performance-only measurement of service quality: a replication and extension. Journal of Bussines Research, 55, 17-31. 
Blázquez, A.; Feu, S. (2010). Sistema de codificación para el análisis de los indicadores de calidad de las cartas de servicios en materia deportiva. Revista Internacional de Ciencias del Deporte. 19(6), 112-127. http://www.cafyd.com/REVISTA/01902.pdf

Calabuig, F.; Saura, D. (1999). La Satisfacción con las instalaciones como herramienta para la gestión deportiva. En D. Ayora y J. E. Gallach (Eds.). Libro de Actas I Congreso sobre la Actividad Física y el Deporte en la Universidad. Valencia: Dpto. Educación Física y Deportes, Universitat de València.

Calabuig, F.; Quintanilla, I., y Mundina J. (2008). La calidad percibida de los servicios deportivos: diferencias según instalación, género, edad y tipo de usuario en según instalación, género, edad y tipo de usuario en servicios náuticos. Revista Internacional de Ciencias del Deporte 4(10), 25-43.

Carman, J. M. (1990). Consumer Perceptions of service quality: An assessment of the SERVQUAL dimensions. J ournal of Retailing, 66, 33-55.

Carrasco, D.; Buendía, D.; Navarro, A.; Valencia, M. J., y Llorente, V. (2006). La evaluación de la eficiencia de los servicios públicos deportivos a través de modelos de cálculo de costes e indicadores de gestión. Revista de Administração e Contabilidade da Unisinos, 3(3), 311-320

Castellano, J.; Hernández, A.; Gómez de Segura, P.; Fontetxa, E., y Bueno, I. (2000). Sistema de codificación y análisis de la calidad del dato en el fútbol de rendimiento. Psicothema 12(4), 635-641.

Consejo Superior de Deportes (2005). Censo Nacional de Instalaciones Deportivas. Madrid: editorial CSD.

Delgado, M. A.; del Villar, F. (1994). El análisis de contenido en la investigación de la enseñanza de la educación física. Revista de Motricidad, 1, 25-44.

Dorado, A.; Gallardo, L. (2003). La incidencia en la valoración de la calidad de un servicio deportivo en función de los factores que determinan la prestación del mismo. En Las Ciencias de La Actividad Física y el Deporte en el Marco de la Convergencia Europea. Actas del III Congreso de la Asociación Española de Ciencias del Deporte. Valencia: Asociación Española de Ciencias del Deporte. Obtenido el 5 de marzo desde http://www.unex.es/eweb/cienciadeporte/congreso/04\%20val/pdf/c162.pdf

Escobar, B.; González J. M. (2007). Reingeniería de procesos de negocio: análisis y discusión de factores críticos a través de un estudio de caso. Revista Europea de Dirección y Económia de la empresa, 16(3), 93-114.

Gallardo, L.; Jiménez, A. (2004). La gestión de los servicios deportivos municipales: vías para la excelencia. Barcelona: INDE.

Gambau, V. (2004). La gestión de la actividad física y deporte para los mayores: ¿cómo evitar el abandono de la práctica deportiva?. En B. Mariño, J. M. Cancela, F. Camiña, J.M. Pazos. Actividad física y deporte para mayores (coords). Actas del IV Congreso Internacional de A. F. y Deporte para Mayores (pp.75-90). La Coruña: Diputación de A Coruña.

García de Yébenes, M. J.; Rodríguez, F., y Carmona, L. (2009). Validación de Cuestionarios. Reumatología clínica, 5(4), 171-177.

García, S. (2007). Los recursos humanos aplicados a la gestión deportiva. Barcelona: INDE.

Goetz, J. P.; Lecompte, M. D. (1988). Etnografía y diseño cualitativo en investigación educativa. Madrid: Morata.

González, M. (2004). Gestión deportiva, tres ejes de calidad, más allá de las personas y los gobiernos. Educación Física Chile, 263, 38-47.

Gorospe, G.; Hernández, A.; Anguera, M. T., y Martínez de Santos, R. (2005). Desarrollo y optimización de una herramienta observacional en el tenis de individuales. Psicothema 17(1), 123-127. 
Blázquez, A.; Feu, S. (2010). Sistema de codificación para el análisis de los indicadores de calidad de las cartas de servicios en materia deportiva. Revista Internacional de Ciencias del Deporte. 19(6), 112-127. http://www.cafyd.com/REVISTA/01902.pdf

Grönroos, C. (1994). Marketing y gestión de servicios. La gestión de los momentos de la verdad y la competencia en los servicios. Madrid: Ed. Díaz de Santos.

Guba, E. (1981). Criterios de credibilidad en la investigación naturalista. En J. Gimeno y A. Pérez Gómez (Eds.). La enseñanza: su teoría y su práctica (pp. 148-165). Madrid: Akal.

Heras, M. (1996). Gestión de la producción. Barcelona: ESADE.

Hernández Mendo, A. (2001). Un cuestionario para evaluar la calidad en programas de actividad física. Revista de Psicología del Deporte, 10(2), 179-196.

Horovitz, J. (1992). La calidad del servicio. Madrid: McGraw-Hill.

Instituto Nacional de Seguridad e Higiene en el trabajo (2008). NTP54: Documentos: Definiciones. Obtenido el 1 de julio de 2009 desde http://www.insht.es/InshtWeb/Contenidos/Documentacion/FichasTecnicas/NTP/Fichero s/001a100/ntp_054.pdf

Jiménez, M.A.; Quesada, F.J.; Aranguren, J., y Ortega, S. (2006). La gestión económica y financiera en una organización deportiva. En J. Lozano, y L. Gallardo (coords.) Futuras claves en la gestión de organizaciones deportivas (pp. 33-50). Cuenca: Universidad de Castilla-La Mancha.

Junta de Andalucía (2004). Manual de elaboración de cartas de servicios. Sevilla: Consejería de Gobernación. Junta de Andalucía. Obtenido el 9 de junio de 2009 desde http://www.juntadeandalucia.es/gobernacion/opencms/portal/com/bin/portal/Administ racionLocal/Publicaciones/manual_cartas_servicio/cartas_servicios.pdf.

Kim, D.; Kim, S. (1995). QUESC: An instrument for assessing the service quality of sport centres in Korea. J ournal of Sport Management, 9, 208-220.

Krippendorff, K. (1990). Metodología de análisis de contenido. Teoría y práctica. Barcelona: Paidós.

Llorens, F. J.; Fuentes, M. (2000). Calidad total. Fundamentos e implantación. Madrid: Pirámide.

López, A. (2001). La formación del gerente público del deporte. Barcelona. Apunts: Educación física y deportes, 66, 64-69.

Luna-Arocas, R. (1998). Segmentación psicográfica y marketing deportivo. Revista de psicología del deporte, 7 (13), 121-134.

Luna-Arocas, R.; Mundina, J. (1998). La satisfacción del consumidor en el marketing del deporte. Revista de psicología del deporte, 7(13), 147-156.

Mancomunidades Integrales de Municipios de Extremadura (2008). Cartas de Servicios. Obtenido el 5 de mayo de 2009 de http://www.desarrolloruralextremadura.com/administracion_local/administracion_local /planes_estrategicos_mancomunidades/cartas

Mañas, M. A.; Giménez, G.; Muyor, J. M.; Martínez, V., y Moliner, C. P. (2008). Los tangibles como predictores de la satisfacción del usuario en servicios deportivos. Psicothema 20(2), 243-248.

Medina, J. (1996). Proceso de entrenamiento de codificadores para el estudio de los diarios del profesorado de educación física. Revista Motricidad, 2, 113-127.

Morales, V.; Hernández-Mendo, A., y Blanco, A. (2005). Evaluación de la calidad en los programas de actividad física. Psicothema 17( 2), 311-317. 
Blázquez, A.; Feu, S. (2010). Sistema de codificación para el análisis de los indicadores de calidad de las cartas de servicios en materia deportiva. Revista Internacional de Ciencias del Deporte. 19(6), 112-127. http://www.cafyd.com/REVISTA/01902.pdf

Moreno, M. P.; Santos, J. A.; Ramos, L. A.; Sanz, D.; Fuentes, J. P., y Del Villar, F. (2002). Aplicación de un sistema de codificación para el análisis de contenido de la conducta verbal del entrenador de Voleibol. Revista Motricidad, 9, 119-140.

Mundina, J.; Calabuig, F. (1999). El marketing social al servicio de la gestión de calidad: El caso de los servicios públicos náuticos de la Generalitat Valenciana. Apunts: Educación Física y Deportes, (57), 77-83.

Palacios, J. L.; Fulgueiras, F. J., y Catalina, C. (2002). La medición de la calidad en la administración local: el caso de los servicios socioculturales del ayuntamiento de Alcobendas. Dirección y organización: Revista de dirección, organización y administración de empresas, 28, 147-158.

Real Decreto 951/2005, de 29 julio, por el que se establece el marco general para la mejora de la calidad en la Administración General del Estado.

Rodríguez, I.; Agudo, A.; García, M. M., y Herrero, A. (2003). Análisis de los factores determinantes de la calidad percibida en los espectáculos deportivos: Aplicación al fútbol profesional. En Actas del Congreso Mundial de Gestión deportiva. Barcelona: Escuela de Administración de Empresas. Universitat Politècnica de Catalunya.

Teas, R. K. (1993). Expectations, performance, evaluation, and consumers perceptions of quality, Journal of Marketing, 57(4), 18-34.

Zeithmal,V. A.; Parasuraman, A., y Berry, L. (1993). Calidad total en la gestión de servicios. Cómo lograr el equilibrio entre las percepciones y las expectativas de los consumidores. Madrid: Díaz de Santos. 